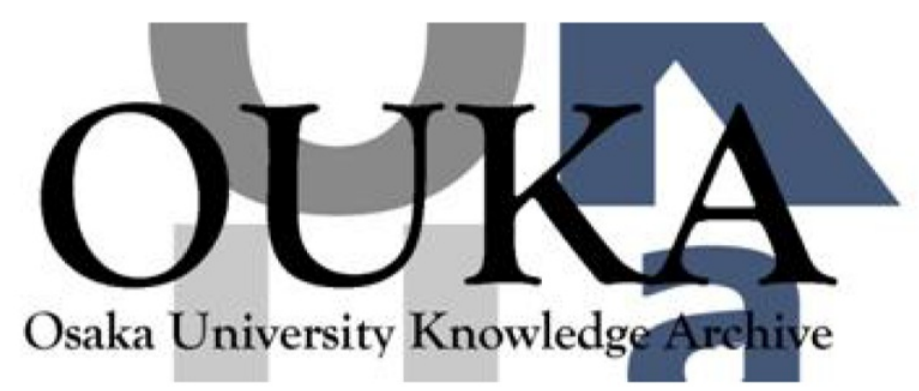

\begin{tabular}{|c|l|}
\hline Title & $\begin{array}{l}\text { Electromechanical effect in freely suspended } \\
\text { liquid crystal films }\end{array}$ \\
\hline Author(s) & $\begin{array}{l}\text { Yablonskii, Sergue i V.; Oue, Toshiyasu; Nambu, } \\
\text { Hidetaka et al. }\end{array}$ \\
\hline Citation & Applied Physics Letters. 75(1) p.64-p.66 \\
\hline Issue Date & $1999-06-05$ \\
\hline oaire:version VoR \\
\hline URL & https://hdl. handle.net/11094/75839 \\
\hline rights & \\
\hline Note & \\
\hline
\end{tabular}

Osaka University Knowledge Archive : OUKA

https://ir. Library. osaka-u. ac. jp/

Osaka University 


\section{Electromechanical effect in freely suspended liquid crystal films}

Cite as: Appl. Phys. Lett. 75, 64 (1999); https://doi.org/10.1063/1.124325

Submitted: 07 December 1998 . Accepted: 11 May 1999. Published Online: 29 June 1999

Serguei V. Yablonskii, Toshiyasu Oue, Hidetaka Nambu, Aleksey S. Mikhailov, Masanori Ozaki, and Katsumi Yoshino

\section{ARTICLES YOU MAY BE INTERESTED IN}

Freely suspended liquid crystal film transfer: A new method of creating thin smectic films on solid substrates

Applied Physics Letters 59, 917 (1991); https://doi.org/10.1063/1.106300

Molecular reorientation and deformation of a freely suspended ferroelectric liquid crystal film Applied Physics Letters 74, 117 (1999); https://doi.org/10.1063/1.122969

Mechanical vibration of freely suspended ferroelectric liquid-crystal film excited by sound and electric field

Journal of Applied Physics 82, 2791 (1997); https://doi.org/10.1063/1.366162

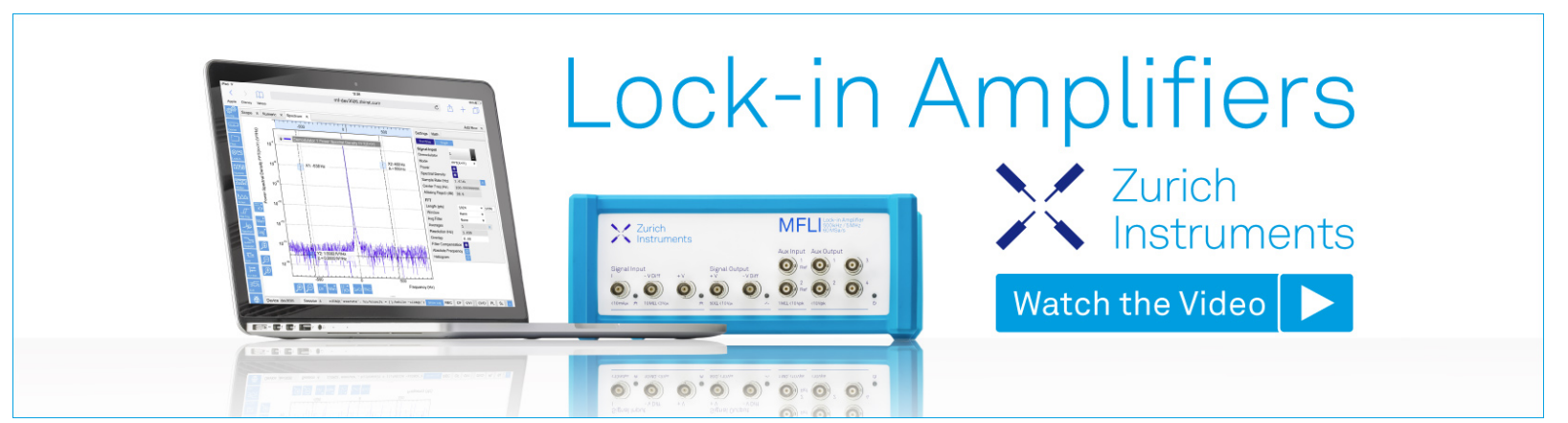




\title{
Electromechanical effect in freely suspended liquid crystal films
}

\author{
Serguei V. Yablonskii ${ }^{\text {a) }}$ \\ Department of Electronic Engineering, Graduate School of Engineering, Osaka University, \\ 2-1 Yamada-Oka, Suita, Osaka 565-0871, Japan \\ Toshiyasu Oue and Hidetaka Nambu \\ Department of Electronic Engineering, Graduate School of Engineering, Osaka University, \\ 2-1 Yamada-Oka, Suita, Osaka 565-0871, Japan \\ Aleksey S. Mikhailov \\ Institute Crystallography of RAS, 117333, Leninskii pr. 59, Moscow, Russia \\ Masanori Ozaki and Katsumi Yoshino ${ }^{\text {b) }}$ \\ Department of Electronic Engineering, Graduate School of Engineering, Osaka University, \\ 2-1 Yamada-Oka, Suita, Osaka 565-0871, Japan
}

(Received 7 December 1998; accepted for publication 11 May 1999)

\begin{abstract}
Electromechanical effect in a freely suspended film based on 4- $n$-octyl-4' - cyanobiphenyl has been detected by means of light scattering at both the first and second harmonics of the exciting electric field. Experimental studies on voltage and frequency dependencies of the optical response are carried out. Organic solvent vapor is shown to strongly affect the electromechanical response. A feasible mechanism for the electromechanical effect is discussed. (C) 1999 American Institute of Physics. [S0003-6951(99)04327-2]
\end{abstract}

Liquid crystals (LC) have attracted considerable attention because of the wide spectrum of their electro-optical effects. ${ }^{1}$ Parallel with the study on the electro-optical phenomena, the electromechanical (EM) effect in the liquid crystalline phases has also attracted great interest from the fundamental and practical points of view. Similar effects have been investigated in detail in various monomer and polymer ferroelectric liquid crystals $(\mathrm{FLC})^{2-6}$ in which an alternating electrical field coupled with spontaneous and flexoelectric polarizations gives rise to a shear deformation of LC accompanying a mechanical vibration of the confining glassy plates.

The EM effect in two-dimensional liquid crystalline systems based on smectic membranes known as freely suspended (FS) film ${ }^{7}$ has been of a special interest. The mechanical vibration of the surface of FLC freely suspended film was effectively excited by a lateral alternating electric field and studied by means of light scattering at the frequency of applied electric field. ${ }^{8-10}$ Recently, a linear EM effect has also been detected in a bilayer lipid membrane. ${ }^{11}$ However, the linear EM effect has never been observed in FS films based on thermotropic achiral LC. In this letter, we report both linear and quadratic EM effects in the FS film based on nonferroelectric mesogen, 4- $n$-octyl-4' -cyanobiphenyl (8CB). For comparison, we also show some results of the EM effect in a FLC freely suspended film.

Measurements on $8 \mathrm{CB}$ which has a smectic A phase between 21 and $33^{\circ} \mathrm{C}$, were carried out at room temperature $T=25^{\circ} \mathrm{C}$. A FS film was prepared across two metal blades. Two polyethyleneterephthalate (PET) sheets were set between the blades. The sample was loaded in the square free area surrounded by the metal blades and PET sheets at room temperature. One of the PET sheets can slide along the blade

\footnotetext{
a) Also at: Institute Crystallography of RAS, 117333, Leninskii pr. 59, Moscow, Russia.

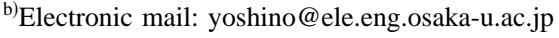

to expand the FS film. These blades were also used as electrodes to apply ac electric field to the FS film. The distance between the electrodes was $3 \mathrm{~mm}$ and the expanded square area was changed from 10 to $30 \mathrm{~mm}^{2}$. For comparison, FLC CS-1029 (Chisso) which exhibits a phase sequence of $-\mathrm{Cr}$ $18^{\circ} \mathrm{C} \mathrm{SmC} * 73^{\circ} \mathrm{C} \mathrm{SmA} 85^{\circ} \mathrm{C} \mathrm{N}^{*} 91^{\circ} \mathrm{C}$ Iso was also used. The experimental setup shown in Fig. 1 enabled us to measure a change of the intensity of light reflected from the surface of the FS film. The film was illuminated by circularly polarized light of a $\mathrm{He}-\mathrm{Ne}$ laser $(632.8 \mathrm{~nm})$. The beam was $1 \mathrm{~mm}$ in diameter. The deformation of the FS film caused a deviation of the reflected beam, which could be sensed by a pinhole detector set at about $400 \mathrm{~mm}$ from the FS film. However, not only deformation but also the change of reflectance due to the molecular reorientation could modulate the optical response. To evaluate this contribution, a photodetector with large aperture was used. Thus, the light could be analyzed by either a photodiode located behind a pinhole of $1 \mathrm{~mm}$ diameter or a photodiode with a $7 \mathrm{~mm}$ circular section aperture. A computer program performed the data acquisition in the

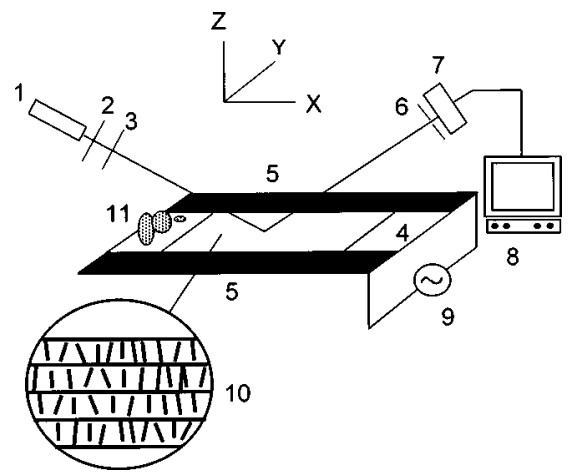

FIG. 1. Experimental setup: (1) He-Ne laser, (2) $\lambda / 4$ plate, (3) Glan polarizer, (4) PET sheet, (5) blades, (6) pinhole, (7) Si photodiode, (8) computer equipped with an analog to digital (A/D) converter or a lock-in amplifier, (9) function generator with broad band amplifier, (10) freely suspended film, and (11) drops of the solvent. 

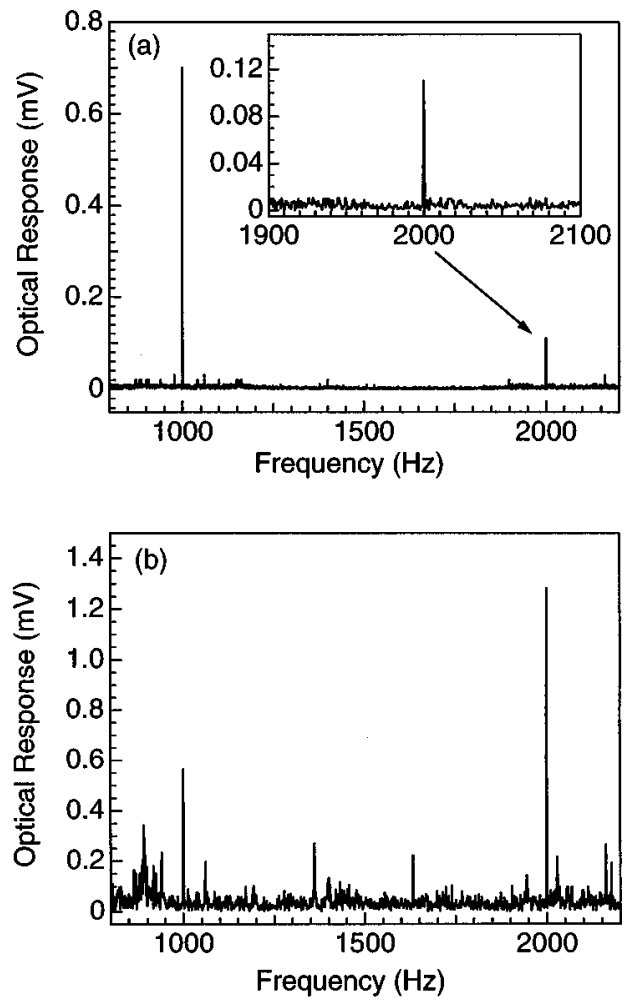

FIG. 2. Fourier spectra of the electro-optical response for: (a) FS thin film and (b) FS thick film. (8CB; SmA phase; alternating voltage with amplitude $U=160 \mathrm{~V}$ was applied to the electrodes; $f=1 \mathrm{kHz}$ ).

range of $10 \mathrm{~Hz}-20 \mathrm{kHz}$ and produced Fourier spectra of scattering intensity. The thickness of the FS film was determined roughly by visual observation of white light reflected by the film. FS films used differed a lot in thickness. For example, thin films had a dark gray color (less than 10 layers ${ }^{12}$ ) and for thick FS films blue color (about 60 layers $^{13}$ ) was observed.

The most basic observation was the appearance of the optical response at the fundamental frequency of an exciting electric field. Figures 2(a) and 2(b) show the Fourier spectra of the EM response induced in thin and thick FS films, respectively. In this case the signal was detected by a pinhole photodetector. In the thin film the second harmonic component of the signal is weaker than the fundamental one, and vice versa for the thick FS film. It should also be noted that the position of the light beam on the surface of the FS film strongly affected the optical response. For example, the

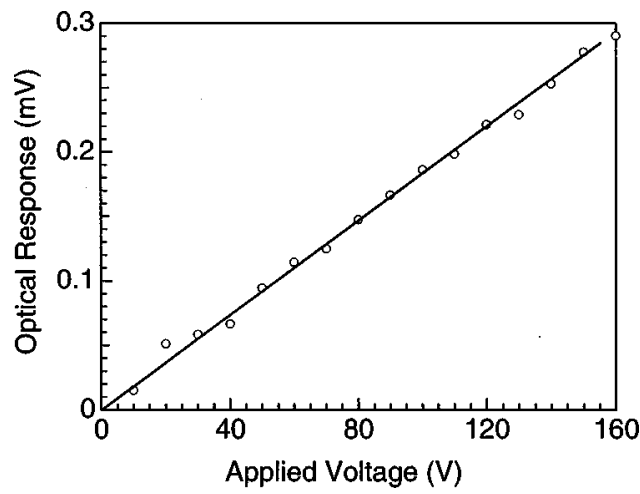

FIG. 3. Typical linear dependence of the optical response at the fundamental frequency as a function of the voltage applied on the electrodes $(8 \mathrm{CB}, \mathrm{SmA}$ phase; $f=1 \mathrm{kHz}$ ).

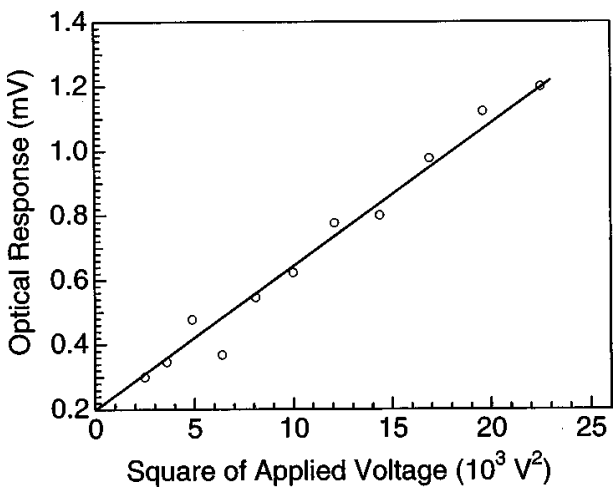

FIG. 4. Typical quadratic voltage dependence of the optical response at the double frequency as function of the voltage applied on the electrodes $(8 \mathrm{CB}$; SmA phase; $1 \mathrm{kHz}$ )

maximum optical response was detected when the laser beam probed the surface of the FS film very close to the electrode, and the signal was not detectable at all when the probe light was reflected from the center of FS film. The signal was absent when the photodetector with the large aperture was used. This proved that the modulation of the reflected beam originated not from the reflectivity change, but from the deformation of the FS film. Figures 3 and 4 show typical observations of the expected linear and quadratic voltage dependence of the first and second harmonics of the optical response. An EM response signal as low as $3 \times 10^{-6} \mathrm{~V}$ was detectable in this experiment and linearity was confirmed over two decades.

The frequency dependence of the linear EM effect for the thin FS film is shown in Fig. 5. The main resonance occurred at $f_{\max } \approx 1190 \mathrm{~Hz}$ with a half-width at $\Delta f$ $\approx 320 \mathrm{~Hz}$. It should be noted that the position of the resonance peak was sensitive to the vapor of the organic solvents. For example, the exposure of the film to the vapor of alcohol from drops of this solvent deposited onto the PET sheet near the FS film as shown in Fig. 1, distinctly (by 30\%) reduced the strength of the optical response.

For comparative analysis the Fourier spectra of the optical response in the FS film based on FLC are shown in Fig. 6. The measurements were carried out at $25^{\circ} \mathrm{C}$ in the $\mathrm{SmC}^{*}$ phase.

Although the SmA liquid crystal belongs to the nonpiezoelectric class $D_{\infty h}$, nevertheless there can be a linear coupling between smectic curvature and external electric field,

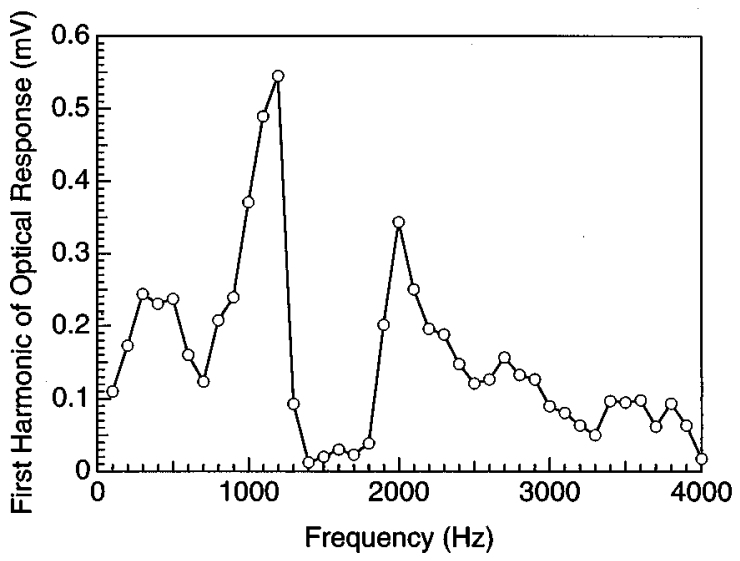

FIG. 5. Frequency dependence of the linear EM effect for FS film based on $8 \mathrm{CB}$ 

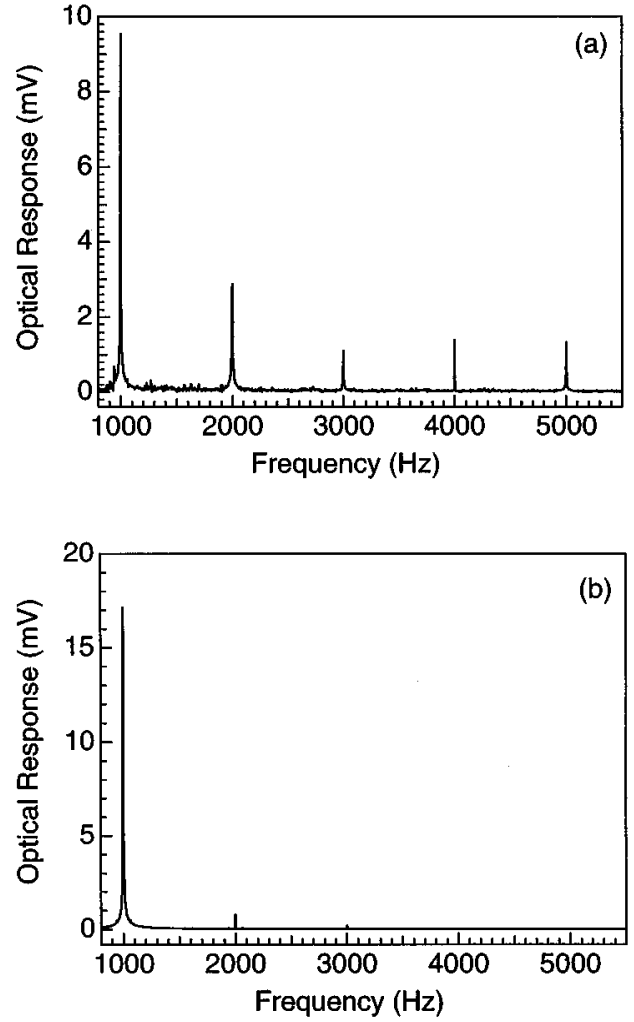

FIG. 6. Fourier spectra of the electro-optical response of thin FS film based on FLC for: (a) $1 \mathrm{~mm}$ pinhole photodetector and (b) $7 \mathrm{~mm}$ aperture photodetector $\left(T=25^{\circ} \mathrm{C}\right)$

i.e., flexoelectric effect. ${ }^{14}$ The lowest-order linear coupling to the electric field $E$ is proportional to the volume torque exerting on the director $\mathbf{n}$ of the LC and has the form of either $E \nabla n$ or $\nabla E n .{ }^{15}$ In our case, both these flexoelectric contributions could induce a linear EM effect, since in the vicinity of the probed region, close to the electrodes, there is a strong electric field as well as its gradient. The probable additional contribution to the linear EM effect proceeds from the static electric field of the diffusion part of the double layer $E_{\text {in }}$ that also adds to the quadratic response of a nematic (or smectic) LC, the linear one. ${ }^{16}$ Indeed, since the dielectric torque is proportional to the total field squared $M_{d} \cong \Delta \epsilon E^{2}$ $=\Delta \epsilon\left(E_{0} \sin \omega t+E_{\text {in }}\right)^{2}$ the cross term corresponds to the "quasilinear" effect with respect to the applied sinusoidal electric field.

The dominant role of the linear EM effect in a thin FS film and quadratic EM effect in a thick film can be interpreted as follows. (i) Both the linear and quadratic effects are developed in the vicinity of wedge-like electrodes at the distance $L \cong h=100 \mu \mathrm{m}$ ( $h$ is the thickness of blade) where a strong inhomogeneous electric field exists. (ii) The quasilinear effect occurs within the Debye screening length $L_{D}$ (for the usual concentration of impurity $L_{D}<1 \mu \mathrm{m}$ ). ${ }^{1}$ (iii) The penetration length of the deformation due to the surface flexoelectric effect for the nematic-like mode is equal to $L_{F}$ $=\left(8 \pi K_{11} / \nu \gamma\right)^{1 / 2} \cong 1.5 \mu \mathrm{m} \quad\left(K_{11}=10^{-6} \mathrm{dyn}\right.$ is the elastic constant of $8 \mathrm{CB}, \gamma=1$ is the rotational viscosity, $\nu$ $=1000 \mathrm{~Hz}$ is the frequency of an applied electric field). ${ }^{17}$ Consequently, according to inequality $L \gg L_{D} \cong L_{F}$ the increase of the volume fraction of the FS films will give rise to the essential change (for our case increase) of the dielectric contribution only and prevailing of the second harmonic in the optical response.

Symmetry breaking near the surface of the FS film may also play a role in the linear EM response. Namely, near the surface the bulk inversion symmetry breaks and surface polarization should be induced, which results in the linear coupling of the polarization and electric field. In our case, however, EM response can be observed only in the vicinity of the electrodes and the surface effect is not dominant.

The most probable reason why the alcohol vapor influenced the EM effect, consists in the change of the surface tension of the FS film. According the Rayleigh theory the eigenfrequency of membrane is proportional to the square root of the surface tension. ${ }^{7}$ Thus, assuming the typical value of surface tension of the smectic membrane $\tau \cong 50 \mathrm{dyn} / \mathrm{cm},{ }^{7}$ the position of the resonance peak $\nu_{\max }=1190 \mathrm{~Hz}$ and the shift of the frequency $\Delta \nu=170 \mathrm{~Hz}$, it is possible to evaluate the value of the surface tension to be $\tau_{1}=38 \mathrm{dyn} / \mathrm{cm}$ after the exposure of the FS film to the solvent vapors.

Fourier spectra of the EM effect in the FLC FS films presented in Fig. 6 manifest clearly defined nonlinear properties due to both the mechanical vibration and the change of the Fresnel coefficients. It seems likely that the strong coupling of the spontaneous polarization of the FLC with the electric field results in a complicated nonsinusoidal surface relief of the FS film.

To summarize, we have presented an experiment on the linear EM effect in the FS films based on $8 \mathrm{CB}$. The origin of the linear EM effect has been discussed. We believe that the influence of the solvent vapor on the EM effect can be used for the detection of small amount of volatile material.

This work was supported by Grant-in-Aid for Scientific Research from the Ministry of Education, Science, Sports, and Culture of Japan and COPERNICUS (IC15-CT96-0744), INTAS 95IN-RU-0128, Russian Fund for Fundamental Research (98-021771) Grants.

${ }^{1}$ L. M. Blinov, Electro-Optical and Magneto-Optical Properties of Liquid Crystal (Wiley, New York, 1983).

${ }^{2}$ A. Jakli, L. Bata, A. Buka, N. Eber, and I. Janossy, J. Phys. (France) Lett. 46, L-759 (1985).

${ }^{3}$ A. Jakli, L. Bata, A. Buka, and N. Eber, Ferroelectrics 69, 153 (1986).

${ }^{4}$ G. Por and A. Buka, J. Phys. 50, 783 (1989).

${ }^{5}$ A. Jakli and A. Saupe, Liq. Cryst. 9, 519 (1991).

${ }^{6}$ N. Eber, L. Bata, G. Scherowsky, A. Schlwa, Ferroelectrics 122, 139 (1991)

${ }^{7}$ P. Pieranski, L. Beliars, J.-Ph. Tournellec, X. Leoncini, C. Furtlehner, H. Dumoulin, E. Riou, B. Jouvin, J.-P. Fenerol, Ph. Palaric, J. Heuving, B. Cartier, and I. Kraus, Physica A 194, 364 (1993).

${ }^{8}$ E. I. Demikhov, S. A. Pikin, and E. S. Pikina, Phys. Rev. E 52, 6250 (1995).

${ }^{9}$ S. Uto, E. Tazoh, M. Ozaki, and K. Yoshino, J. Appl. Phys. 82, 2791 (1997).

${ }^{10}$ S. Uto, M. Ozaki, and K. Yoshino, Appl. Phys. Lett. 74, 117 (1999).

${ }^{11}$ A. T. Todorov, A. G. Petrov, and J. H. Fendler, J. Phys. Chem. 98, 3076 (1994).

${ }^{12}$ Ch. Bahr and D. Fliegner, Phys. Rev. A 46, 7657 (1992).

${ }^{13}$ E. B. Sirota, P. S. Pershan, L. B. Sorensen, and J. Collett, Phys. Rev. A 36, 2890 (1987).

${ }^{14}$ P. G. de Gennes and J. Prost, The Physics of Liquid Crystals (Clarendon, Oxford, 1993).

${ }^{15}$ J. Prost and P. S. Pershan, J. Appl. Phys. 47, 2298 (1976).

${ }^{16}$ L. M. Blinov, S. P. Palto, D. G. Sikharulidze, and S. V. Yakovlev, Appl. Phys. Lett. 72, 3377 (1998).

${ }^{17}$ S. V. Yablonskii, M. Rajteri, C. Oldano, and G. Durand, Proc. SPIE 2731, 87 (1996). 